\title{
Perforation of the pulmonary artery requiring pneumonectomy after the use of a flow-directed (Swan-Ganz) catheter
}

\author{
MIECRYLOW M DEREN, PAUL G BARASH, GRAEME L HAMMOND, \\ AND TEODORO SAIEH
}

From the Department of Surgery and Anesthesia, Yale Medical School, and the Surgical and Anesthesia Services, Yale-New Haven Hospital, New Haven, Connecticut 06510, USA

ABSTRACT A case of pulmonary artery perforation by a flow-directed balloon-tipped catheter necessitated pneumonectomy. Predisposing conditions are discussed and conclusions made on the basis of this case and previous reports.

The flow-directed balloon-tipped catheter has been widely used in cardiac surgical patients (Katz et al, 1977 ) in patients undergoing major surgical procedures and in emergencies for critically ill patients requiring assessment and management for cardiopulmonary deterioration or disturbances of fluid balance or both (Swan and Ganz, 1975).

At Yale these catheters are used routinely in patients undergoing cardiac surgical procedures. Over 2000 such catheters have been inserted resulting in considerable experience in their placement. Immediate complications include arrhythmias, carotid artery puncture, and pneumothorax. Long-term complications include sepsis, pulmonary infarction, and neuropathy (Katz et al, 1977). The common complications associated with their insertion are usually not severe and have not been associated with lethal consequences (Foote et al, 1974; Katz et al, 1977). Major complications have been described, among which are five cases of pulmonary artery perforation, two of them fatal (Chun and Ellestad, 1971; Lapin and Murray, 1972; German et al, 1973; Golden et al, 1973; Page et al, 1974).

\section{Case report}

A 76-year-old woman with a history of progressive congestive heart failure secondary to long-standing aortic stenosis underwent aortic valve replacement. Physical findings were typical for stenotic aortic valvar disease with mild congestive heart failure.

Laboratory reports were normal including the prothrombin time and partial thromboplastin time. The chest radiograph showed pulmonary vascular congestion and cardiomegaly. Cardiac catheterisation showed a left ventricular enddiastolic pressure of $38 \mathrm{mmHg}$ and a pulmonary artery pressure of $40 / 24 \mathrm{mmHg}$.

Before induction of general anaesthesia, a 7F Swan-Ganz catheter was inserted percutaneously via the right internal jugular vein into the pulmonary artery by a technique we have previously reported (Barash and Dizon, 1977). The catheter insertion was uneventful and cardiac output readings were appropriate.

The patient underwent uneventful aortic valve replacement with a Carpentier-Edwards porcine valve. Cardiopulmonary bypass was discontinued without difficulty, cannulae were removed, protamine was administered, and preparations for placement of sternal wires were made.

At this time the patient became acutely hypotensive. She was immediately given heparin and cardiopulmonary bypass was reinstituted for haemodynamic stabilisation. After stabilisation was obtained, a bulging of the right pleura was noted. Further examination of the right chest showed about 21 of clotted and unclotted blood in the pleural space. The tip of the flow-directed catheter could easily be seen perforating the pulmonary artery in the region of the hilum between upper and middle lobes. The catheter was initially noted to be partially inflated.

Attempts to repair this laceration with sutures and Teflon pledgets during cardiopulmonary bypass were at first successful. With termination of 
cardiopulmonary bypass and restoration of mechanical ventilation, however, persistent severe haemorrhage occurred. This necessitated a right pnumonectomy performed without bypass through the median sternotomy.

Oxygenation appeared adequate after this operation, and the patient was transferred to the intensive care unit with vasopressor support. After an initial period of cardiac instability immediately after the operation, haemodynamic stabilisation was achieved but a tracheostomy was required for continued artificial ventilation. Two weeks after operation the patient was alert and doing reasonably well but still required assisted ventilation. Three and a half weeks after operation the patient began to deteriorate and died from renal failure, gastrointestinal bleeding, low cardiac output, central nervous system dysfunction, and respiratory failure.

Pathological examination of the specimen of the right lung showed characteristic changes of pulmonary hypertension with a tear in the pulmonary artery in the hilum at the junction of the right upper and middle lobes.

\section{Comment}

Although five previous cases of pulmonary artery perforation have been reported (Chun and Ellestad, 1971; Lapin et al, 1972; German et al, 1973; Golden et al, 1973; Page et al, 1974), the factors involved in these perforations by balloontipped catheters are unclear. Several predisposing factors have been emphasised; pulmonary hypertension (German et al, 1973), an excessively peripheral location of the catheter (Chun and Ellestad, 1971), a large loop of catheter in the right atrium or right ventricle which may allow the tip to perforate the pulmonary wall secondary to catheter motion (Chun and Ellestad, 1971), and anticoagulation (Golden et al, 1973).

Another factor is the technique for positioning the catheter tip in the pulmonary vessels (Chun and Ellestad, 1971; Golden et al, 1973). Swan and Ganz in their original paper (Swan et al, 1970) recommended that the balloon be deflated in the wedge position, advanced $1-3 \mathrm{~cm}$, and then reinflated each time the wedge pressure measurement was required. In contrast to this, Lapin and Murray (1972) suggested that when the balloon is inflated in the wedge position it creates a pressure gradient across the pulmonary artery wall that may lead to perforation. They recommended inflating the balloon in place each time a wedge pressure recording is needed. Presumably the method of Lapin and Murray would decrease the incidence of the rare case of peripheral pulmonary artery perforation.

In our patient the balloon-tipped catheter was placed using a method similar to that suggested by Lapin and others that should theoretically minimise the danger of pulmonary artery damage. Of the previously reported cases of fatal perforation, one was peripheral and the second was more central. Our patient's perforation was definitely central in location. This suggests that leaving the balloon-tipped catheter in a central location and not advancing it $1-3 \mathrm{~cm}$ as originally suggested by Swan and Ganz may still leave the patient at risk for a laceration of the pulmonary artery at the hilum rather than peripherally, a far more hazardous situation than when the catheter is peripheral and perforation occurs.

Other mechanisms for perforation of the pulmonary artery in the hilar area in our patient may be speculated:

(1) The fragility of the tissues in this 76-year-old patient may have been a factor in causing perforation as well as creating difficulty in achieving adequate repair once the laceration had occurred.

(2) The pulmonary hypertensive changes noted on pathological examination of the pulmonary artery may also have contributed to the tear (Lemen et al, 1975). In the previously described cases pulmonary hypertension was moderately severe in one case (Golden et al, 1973) and mild in another (Lapin and Murray, 1972). In neither was it correlated per se with the occurrence of the perforation of the pulmonary artery.

(3) Although our patient was not given anticoagulants before operation, she had recently received heparin for cardiopulmonary bypass and then protamine reversal. The possibility of defective clotting factors and heparin rebound has been reported as a factor in promoting haemorrhage in patients with this complication. This does not, however, appear to be a factor in our case.

(4) Although balloon-tipped catheters are routinely kept deflated on our service, the balloon appeared partially inflated when the laceration was discovered. We do not, however, feel that this caused the laceration since before the perforation there were no signs of persistent wedging or "overwedging" and a good pulmonary artery trace was observed. There was no evidence for malfunction of the catheter itself when it was subsequently removed and examined. This does lead to the speculation that these balloons do not deflate completely unless a negative pressure is applied.

(5) Lemen et al (1975) showed, in three of six dogs with artificially raised pulmonary artery 
pressures, small tears in the pulmonary artery in the area of the catheter tip. These tears were not found in dogs with normal pulmonary artery pressures.

They also showed that inflation of the balloon in a rigid tube caused the catheter tip to deviate into the wall. Accordingly, they postulated that the "inflation of the Swan-Ganz balloon in these distended and less compliant vessels caused the tip to protrude into the wall and that the shearing effect of the tip against the wall, promoted by the transmitted cardiac pulsation, may have produced erosion or actual perforation of the vessel wall."

(6) The potentially high pressure of the inflated catheter balloon can also contribute to such damage. Pressures were measured postoperatively after inflating the balloon with $0.5 \mathrm{ml}, 1 \mathrm{ml}$, and $1.5 \mathrm{ml}$ of air. Measurements were performed in five patients, each one taken three times and then averaged. In each instance the pressure in the balloon was raised above $180 \mathrm{mmHg}$. Pulmonary artery and pulmonary capillary wedge pressures were simultaneously recorded to ensure adequatc functioning of the catheter. The SwanGanz catheter was radiologically in good position, and in each case there was an excellent pulmonary artery trace and wedge trace without "overwedging." A summary of pressure measurements is given in the table.

Swan-Ganz balloon inflation pressures in relation to inflation volume

\begin{tabular}{lccccc}
\hline & $0.5 \mathrm{ml}$ & $1.0 \mathrm{ml}$ & $1.5 \mathrm{ml}$ & $P A$ & $P C W P$ \\
\hline $\begin{array}{c}\text { Mean in } \\
\text { mmHg }\end{array}$ & 181.66 & 231.40 & 259.70 & 21.20 & 14.40 \\
$\begin{array}{c}\text { Standard } \\
\text { error of } \\
\text { mean }\end{array}$ & 6.67 & 12.40 & 18.97 & 1.29 & 1.41 \\
\hline
\end{tabular}

PA = Pulmonary artery.

$\mathrm{PCWP}=$ Pulmonary capillary wedge pressure.

In each case the pressure was raised to levels similar to those reported in the high pressure cuffs of endotracheal tubes. The exact pressure exerted by the balloon on pulmonary vessel walls is, at present, unknown but would probably be high and the trauma to small arterioles significant. The role of this high pressure balloon is speculative and warrants further study and possible redesign of the balloon-tip catheter.

\section{Conclusions}

Whether the tip of the Swan-Ganz catheter is positioned centrally in the right or left main pul- monary artery or whether it is positioned more peripherally, the possibility of perforation is present. From published reports, this is fortunately a rare complication. As cur case illustrates, however, its consequences are more serious if it occurs centrally. Although the possibility of damage to the pulmonary artery in the hilum is normally rare, it may be more likely in patients with frail tissues and pulmonary hypertension.

Awareness of this complication may assist in making the diagnosis and taking immediate corrective action. It is unlikely that a patient would survive in a ward after a large perforation of the pulmonary artery. The perforation in our case occurred in the operating room with cardiopulmonary bypass readily available for corrective action.

Redesigning the catheter may decrease the possibility of this complication occurring. Design features might include: (1) an inflate-deflate mechanism that prevents over-inflation and allows for monitoring the degree of deflation, and (2) a soft balloon that, when inflated, will conform to the walls of the pulmonary artery rather than stretch it, such as the cuff of a low pressure tracheostomy tube.

Because we obtain useful data from flowdirected balloon-tipped catheters in managing our acutely ill patients, we believe that continued use of such a monitoring device is warranted but that the safety of the catheter could be improved.

\section{References}

Barash, P G, and Dizon, C T (1977). An introducer for intraoperative percutaneous insertion of a SwanGanz catheter. Anesthesia and Analgesia. Current Researches, 46, 444-446.

Chun, G M H, and Ellestad, H (1971). Perforation of the pulmonary artery by a Swan-Ganz catheter. New England Journal of Medicine, 284, 10411042.

Foote, G A, Schabel, S I, and Hodges, M (1974) Pulmonary complications of the flow-directed balloon-tipped catheter. New England Journal of Medicine, 290, 927-931.

German, J C, Allyn, P A, and Bartlett, R H (1973). Pulmonary artery pressure monitoring in acute burn management. Archives of Surgery, 106, 788791.

Golden, M S, Pinder, T, Anderson, W T, and Cheitlin, M D (1973). Fatal pulmonary hemorrhage complicating use of a flow-directed balloon-tipped catheter in a patient receiving anticoagulant therapy. American Journal of Cardiology, 32, 865867.

Katz, J D, Cronau, L H, Barash, P G, and Mandel, $S$ (1977). Pulmonary artery flow-guided catheters in 
the perioperative period, indications and complications. Journal of the American Medical Association, 237, 2832-2834.

Lapin, E S, and Murray, J A (1972). Hemoptysis with flow-directed cardiac catheterisation. Journal of the American Medical Association, 220, 1246.

Lemen, R, Jones, J G, and Cowan, G (1975). A mechanism of pulmonary-artery perforation by Swan-Ganz catheters. New England Journal of Medicine, 292, 211-212.

Page, D W, Teres, D, and Hartshorn, J W (1974). Fatal hemorrhage from Swan-Ganz catheter. New England Journal of Medicine, 291, 260.
Swan, H J C, and Ganz, W (1975). Use of the balloon flotation catheters in critically ill patients. Surgical Clinics of North America, 55, 501-520.

Swan, H J C, Ganz, W, Forrester, J, Marcus, H, Diamond, G, and Chonete, D (1970). Catheterisation of the heart in man with use of a flow-directed balloon-tipped catheter. New England Journal of Medicine, 283, 447-451.

Requests for reprints to: Graeme L Hammond, Yale School of Medicine, 333 Cedar Street, New Haven, Connecticut 06510 . 